\title{
Dielectrophoretically trapping semiconductive carbon nanotube networks
}

\author{
Robert Cicoria and Yu Sun \\ Advanced Micro and Nanosystems Laboratory, University of Toronto, 5 King's College Road, \\ M5S 3G8, Canada \\ E-mail: sun@mie.utoronto.ca
}

Received 6 August 2008, in final form 5 September 2008

Published 11 November 2008

Online at stacks.iop.org/Nano/19/485303

\begin{abstract}
The construction of carbon nanotube field effect transistors (CNT-FETs) can be accomplished by techniques such as spin coating, dielectrophoresis (DEP), and chemical vapor deposition, among which DEP has advantages in terms of the ease of manufacturability and the potential of scalability. In this study, we improved on DEP trapping of CNTs by demonstrating that CNT-FETs of CNT networks with highly semiconductive characteristics are formed with proper tuning of DEP parameters. By investigating the factors that are significant in forming these networks, we rationalize guidelines for effectively fabricating highly semiconductive CNT-FETs with mixtures of metallic and semiconductive multi-walled carbon nanotubes.
\end{abstract}

(Some figures in this article are in colour only in the electronic version)

\section{Introduction}

The carbon nanotube field effect transistor (CNT-FET) has evolved from a bench-top innovation to a promising component in nano electronics. Sensors based upon CNT-FETs are interesting devices because of their fast response [1] and high sensitivity for detecting single molecules in air and liquid [2, 3]. The increasing range of possible sensing applications, initially as gas sensors and later as protein and virus sensors [2] makes them attractive candidates for new generations of sensing systems. Ultimately, ease of device manufacturability and repeatable device characteristics are going to be the defining criteria for determining whether CNTFET based systems can thrive.

Several methods exist for the construction of CNT-FETs. The methods of manipulating individual CNTs with atomic force microscopy [4] or spin coating CNT solution onto a substrate [5, 6] lack speed and control, respectively. Chemical vapor deposition (CVD) is a well adopted technique for growing CNTs in situ to form CNT-FETs [3, 7]. Despite the many attractive aspects of in situ growth, this technique still suffers from catalyst contamination and inability to ensure semiconductive CNTs are grown over undesired metallic CNTs between electrode pairs [6].

Alternatively, CNTs that are synthesized using various approaches (e.g., CVD and arc discharge) can be post-growth manipulated dielectrophoretically. DEP uses predominantly
AC fields to align CNTs in solution on electrodes. It has been used to trap both single-walled and multi-walled nanotubes in both separated single tube forms and network forms [2, 6, 8-10]. FETs with a single CNT spanning the conductance channel are ideal because they function most effectively and are most sensitive [2]. However, single CNT transistors constructed by DEP typically require nanosized electrodes that are e-beam lithography made and have poor reproducibility. Although CNT-FETs with a network of CNTs are reported to be less sensitive than single CNT transistors [2], DEP trapping of CNT networks represents a more reproducible method for forming CNT-FETs. It is quick and inexpensive with recent reports demonstrating parallel FET array fabrication [5, 6].

Although significant efforts have been expended to make CNT-FETs highly semiconductive for sensing applications, the existence of both metallic and semiconductive CNTs in the network impairs their performance. Assuming an even distribution of folding chiralities, $1 / 3$ of all CNTs in a sample should be metallic with the remaining $2 / 3$ exhibiting semiconductive characteristics $[4,11,12]$. In DEP trapping, the presence of both metallic and semiconductive carbon nanotubes in solution is the main reason why forming single nanotube conductance channels is unreliable. The dielectrophoretic forces on CNTs are influenced by the electronic properties of the CNTs and cause a separation of metallic and semiconductive nanotubes [12]. 
At high frequencies, the dielectrophoretic force is proportional to the difference between the dielectric constants of CNTs and the solvent, $F \propto\left(\varepsilon_{p}-\varepsilon_{m}\right) / \varepsilon_{m}$ (4), where $\varepsilon_{p}$ is the dielectric constant of the carbon nanotubes and $\varepsilon_{m}$ is the dielectric constant of the solvent. The force difference is significant because of the high dielectric constant of metallic CNTs (>2000) and the low dielectric constant of semiconductive CNTs (as low as 3) [4, 9]. When a solvent with a dielectric constant between those values is used, separation of CNTs will occur with metallic CNTs attracted to the field source (positive DEP) and semiconductive CNTs repelled (negative DEP) [12]. This physical effect separates CNTs, causing metallic ones to be attracted to field generating electrodes and leaving semiconductive nanotubes in solution $[12,13]$. The attraction of only metallic CNTs to electrodes makes DEP unreliable for the formation of single nanotube CNT-FETs.

When DEP is performed on solutions containing entangled CNT networks of both metallic and semiconductive nanotubes, dielectrophoretic forces tend to draw the bundle to electrodes [12]. Based on the dielectric constants of both types of CNTs and the solution, the dielectrophoretic force on metallic CNTs is approximately two orders of magnitude larger than the force on semiconductive CNTs, $F_{m} / F_{s}=[(2000-$ $\left.\left.\varepsilon_{m}\right) / \varepsilon_{m}\right] /\left[\left(3-\varepsilon_{m}\right) / \varepsilon_{m}\right]=-100$. Thus, the force on metallic CNTs would dominate the cluster [12], and an entangled network containing both types of CNTs in solution will be attracted towards the field emitting electrodes. Therefore, CNT-FETs formed by this process contain both metallic and semiconductive nanotubes. The electrical behavior is highly dependent on the structure of the network. For example, CNT-FETs with smaller networks have been reported to perform well more frequently than those with larger networks [2].

The structure of a trapped CNT network is affected by several factors, such as CNT solution concentration and dispersion, DEP electric field parameters, and trapping time. In this study, we systematically investigate how these factors influence the electronic properties of trapped networks and furthermore, determine effective network forming conditions in order to construct highly semiconductive CNT-FET devices.

\section{Materials and methods}

In experiments, multi-walled CNTs from Nanoledge Inc. with diameters of 70-150 nm were used. The CNTs were suspended in $50 \mathrm{ml}$ of methanol at varying concentrations along with $75 \mu 1$ of Triton $\times 100$ surfactant. Triton $\times 100$ surfactant was chosen because it has been shown to have a minor effect on the electronic properties of carbon nanotubes when adsorbed onto the nanotube surface [14]. The solution was sonicated for three hours immediately prior to trapping to ensure significant although not complete dispersion of CNTs such that small bundles still exist in solution.

DEP trapping was performed on $4 \mu \mathrm{m}$ separated roundedtip Au electrodes on $\mathrm{SiO}_{2}$ coated silicon substrate. A function generator was used to generate both an AC and DC field. The
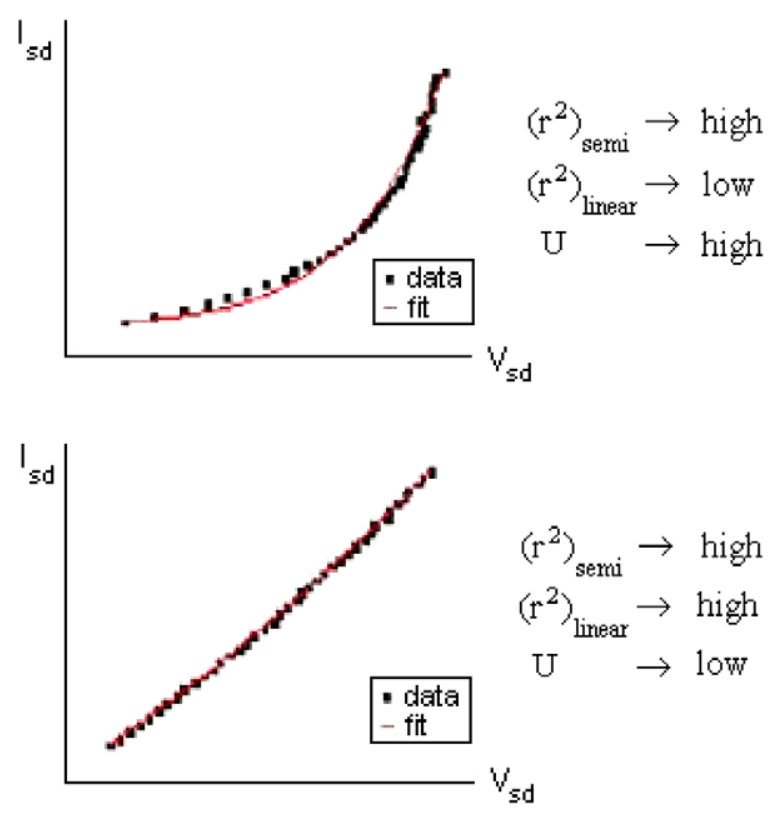

Figure 1. The electronic characteristics of two CNT-FET devices. The metric, $U$, was calculated for each device to quantify the degree of semiconductivity.

varied parameters were AC frequency, AC amplitude, and DC offset. The DC field was added to provide additional force on the nanotubes so that they become bound to the electrodes and orient more favorably [15]. This force acts on charges that develop on the nanotubes from minor reactions in the solvent [16].

When the fields were active, a $6 \mu \mathrm{l}$ drop of the CNT solution was pipetted onto the electrode gap and evaporated within approximately $30 \mathrm{~s}$. The CNT-FETs were characterized by collecting $I-V$ data. The applied current was varied between -4 and $4 \mu \mathrm{A}$, and voltages were recorded.

A second photolithography process was also conducted to deposit a second Au layer to sandwich CNTs on a number of the fabricated CNT-FET devices. This process helped reduce contact resistance [18]. However, comparisons between clamped and non-clamped transistors demonstrated that the impact of the second Au layer produced negligible differences, which confirmed that contact effect was not a concern.

In order to quantify how semiconductive the CNT-FET devices were, we took nonlinearity in the source-drain $I-V$ characteristics as an indicator of semiconductivity [17]. The metric defined for this purpose is $U=r_{\text {semi }}^{2}-r_{\text {linear }}^{2}$, where $r^{2}$ are the coefficients of determination of two mathematical fits (linear and nonlinear) to the data. The nonlinear fit is modeled by $I_{\mathrm{sd}}=a V_{\mathrm{sd}} e^{\mathrm{bVsd}}$, where $a$ and $b$ are the fit parameters. This model was chosen such that nonlinear curves can be well fitted with minimal degrees of freedom in the fit. The metric, $U$, can take a maximum value of one with higher values indicating a more semiconductive behaving network and lower values representing metallic behavior (figure 1). Devices that fail to form under DEP are assigned a value of zero. For this reason, we can take high average utility values to indicate higher semiconductive behavior with high tendency for formation. 


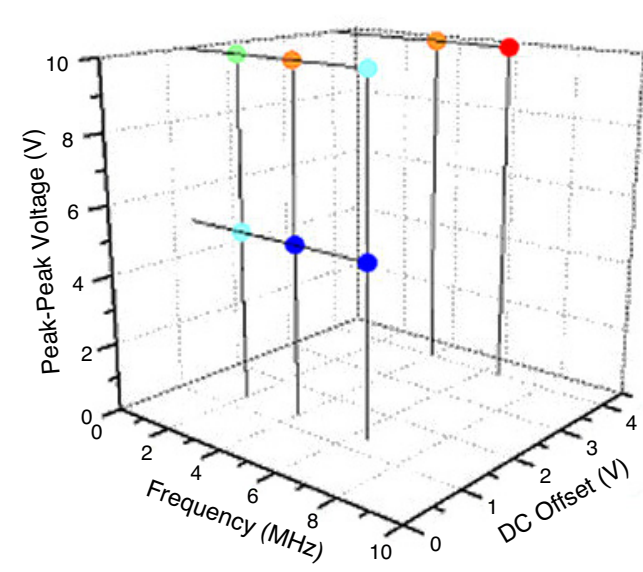

(a)
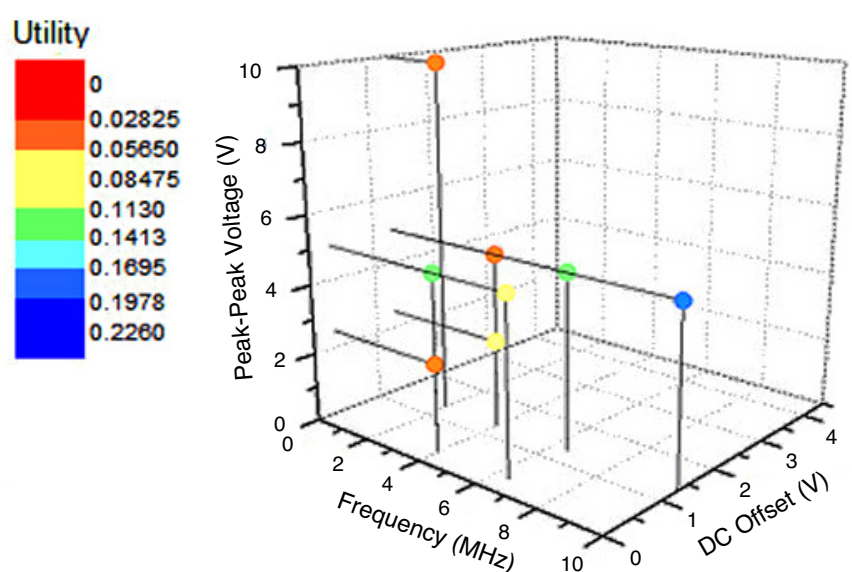

(b)
Utility

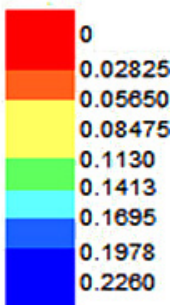

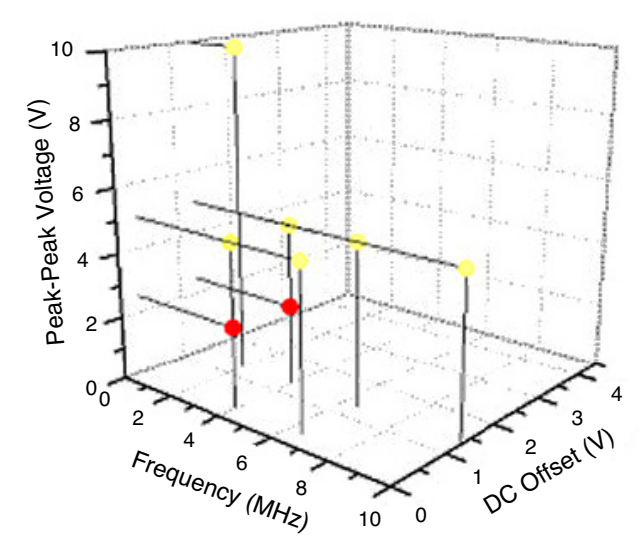

Utility

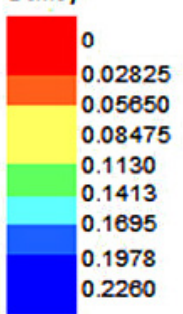

(c)

Figure 2. The semiconductive characteristics of trapped CNT networks in terms of the metric, $U$. Each data point depicts the average value of eight devices for each parameter set. DEP trapping was performed using three different concentrations of CNT solution: (d) $17 \mu \mathrm{g} \mathrm{ml}{ }^{-1}$, (e) $8.9 \mu \mathrm{g} \mathrm{ml}^{-1}$, (f) $4.4 \mu \mathrm{g} \mathrm{ml}^{-1}$.

\section{Results and discussion}

The electronic behavior of DEP trapped CNT networks was quantified under different trapping parameters. We performed DEP trapping using three different concentrations of CNT solution. At each concentration, eight different DEP trapping parameter combinations were used by fabricating eight transistors with each parameter set, resulting in 192 devices that were fabricated and tested. The results are presented using the metric, $U$, and shown in figure 2 . The data depicts the average value of eight devices for each parameter set.

The results show a significant dependence of CNT-FET device characteristics on DEP trapping parameters and solution concentrations. However, conditions exist which consistently produced devices with semiconductive characteristics. The most significant factor is CNT solution concentration. Figure 2 shows that the best devices were fabricated from the highest concentration CNT solution $\left(17 \mu \mathrm{g} \mathrm{ml}^{-1}\right)$. As the solution concentration decreased, the overall device characteristics became more metallic. The trend of FET devices exhibiting more metallic $I-V$ behavior is further elucidated in figure 3 where we show $I-V$ data for three concentrations of CNT solution. The utility values for each device illustrate a gradually decreasing trend in device performance.

We hypothesize that sparse regions within nanotube bundles are responsible for generating the semiconductive behavior of the FET devices. It is unlikely that this effect is due to an increase in the number of semiconductive nanotubes inside the trapped bundles because DEP forces do not favor attracting semiconductive nanotubes to the electrodes. Also, we do not expect that the DEP process is selecting bundles containing a higher number of semiconductive nanotubes from the solution because the purification process utilized in the manufacturing of the CNTs promotes a uniform mixture of nanotubes, ensuring that all large bundles in the solution have the same percentage composition of metallic and semiconductive nanotubes. Our hypothesis that sparse 'bottleneck' regions are responsible for generating the semiconductive characteristics of the FET devices is confirmed via SEM imaging. Figure 4 shows a transistor fabricated at a solution concentration of $17 \mu \mathrm{g} \mathrm{ml}^{-1}$. SEM imaging shows 

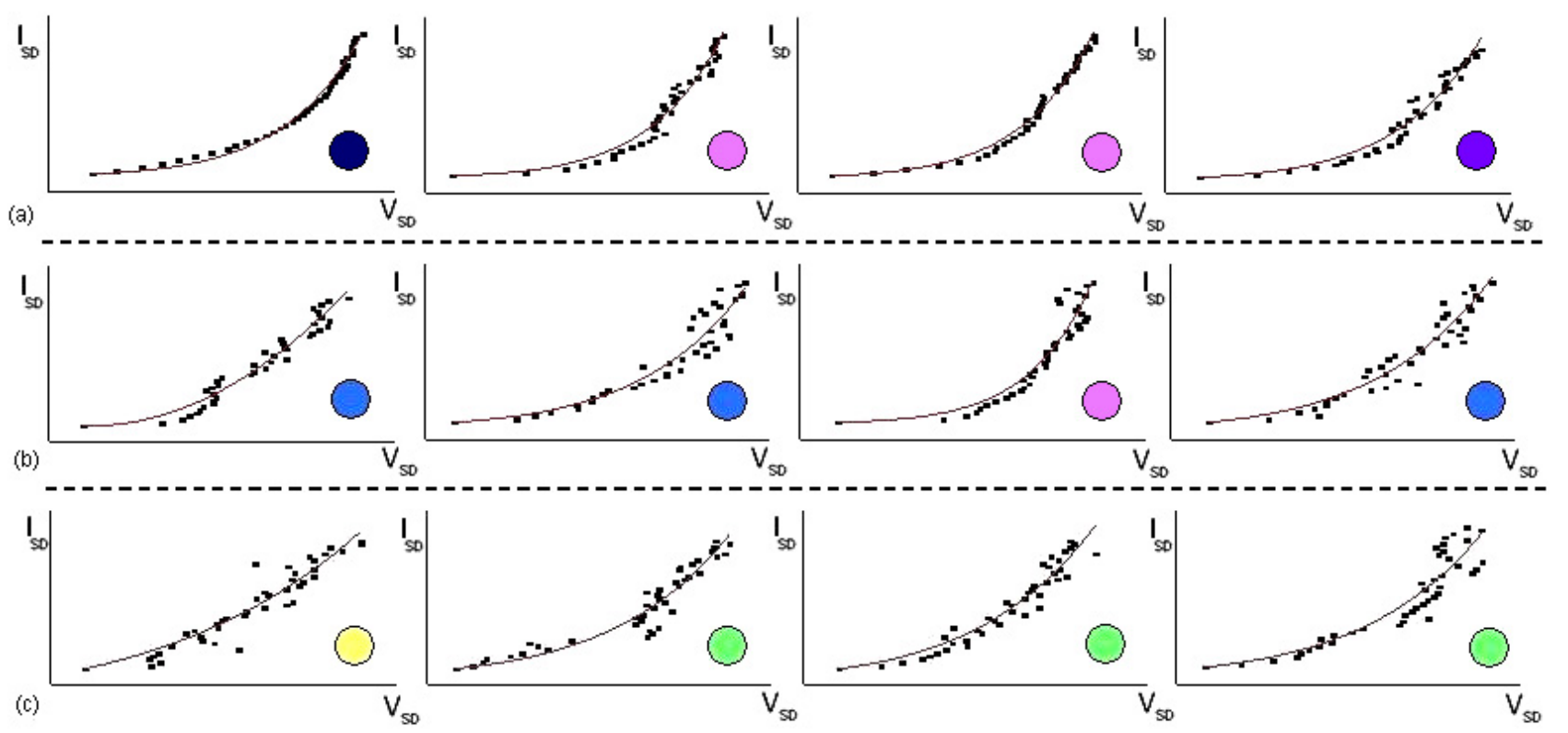

Utility Scale

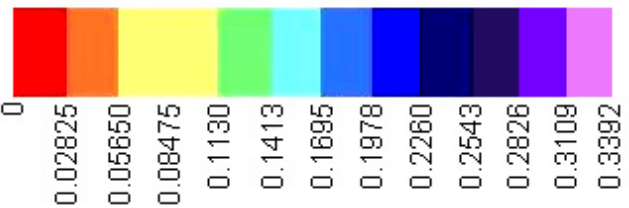

Figure 3. $I-V$ data and fit of representative devices formed at AC $6.5 \mathrm{MHz}, \mathrm{AC}$ peak-peak $5 \mathrm{~V}$ and DC offset $1.3 \mathrm{~V}$. Solution concentration varies: (a) $17 \mu \mathrm{g} \mathrm{ml}^{-1}$, (b) $8.9 \mu \mathrm{g} \mathrm{ml}^{-1}$, (c) $4.4 \mu \mathrm{g} \mathrm{ml}^{-1}$.

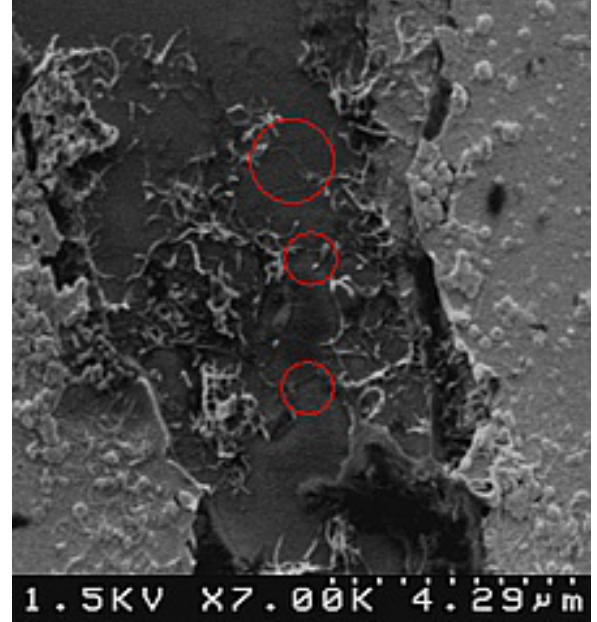

Figure 4. SEM image of a CNT network DEP trapped between two Au electrodes. Red circles indicate regions of potential electronic bottlenecks formed by restricted paths of semiconductive CNTs.

that the network contains dense and sparse regions. In the sparse areas, all current passing through the device is forced through a small number of CNTs. The electrical properties of these CNTs within the transistor channel determine the overall device behavior.

In the hypothetical schematics shown in figure 5, region 1 consists of a dense network of both metallic and
(1) (2) (1)

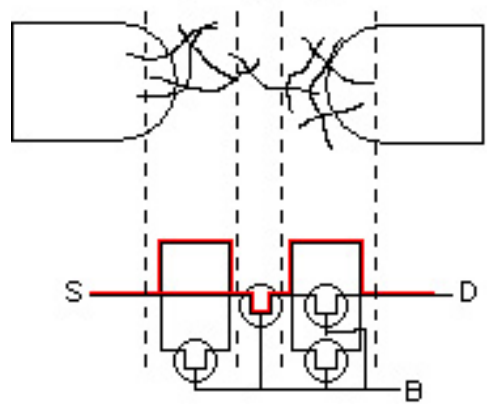

Figure 5. A hypothetical CNT network consisting of metallic and semiconductive CNTs.

semiconductive nanotubes whereas region 2 is a bottleneck area consisting of a sole semiconductive carbon nanotube. In the dense regions, most electrons travel through CNTs of least resistance, namely, through metallic CNTs. The sparse regions are capable of channeling current through semiconductive CNTs forcing an overall semiconductive device response that is dependent on hole transport [19] within the semiconductive nanotubes. Devices that were fabricated with low solution concentrations lack this network structure and instead have metallic CNTs spanning all or most of the electrode gap; this occurs because the bundles of carbon nanotubes in solution 
are smaller. The small bundles have an increased chance of deviating from the solution's overall percentage composition of metallic and semiconductive carbon nanotubes because of their size. In this situation, many small nanotube bundles are attracted to the electrodes, some not containing any semiconductive nanotubes. This promotes the fabrication of a channel containing parallel bridges, thus short circuiting potential bottleneck regions and leading to an overall less semiconductive device behavior.

In addition to CNT concentration, DEP parameter sets also had a significant impact on network trapping. For the multi-walled CNTs used in this study, a specific combination of parameters (AC frequency of 4-10 MHz, DC offset of $1.3 \mathrm{~V}$, and $\mathrm{AC}$ peak-peak voltage of $5 \mathrm{~V}$ ) produced our best trapping results regardless of CNT solution concentration.

Because of differences in CNTs from different manufacturing methods/conditions, it is difficult to extend these exact parameters to other multi-walled CNTs. However, a few observations are worth mentioning. Firstly, at high AC frequencies, DEP trapping was found not very sensitive to differences in frequency, which is consistent with the prediction by Dimaki et al that dielectrophoretic forces will become less dependent on the frequency at high frequencies [4]. Secondly, high intensities of the AC field tended to reduce the efficiency of the process. This observation is in agreement with the observation by Seo et al [20] that the number of deposited nanotube bundles increases with increased AC DEP voltage, which acts to reduce the number of bottleneck regions (sparse regions).

Based on the findings and analysis, we attempt to generate a few guidelines for DEP trapping of multi-walled CNTs to form semiconductive networks. A good starting point would be to use an AC frequency over $4 \mathrm{Mhz}$. Furthermore, the semiconductivity of a trapped network generally decreases as the AC/DC amplitude ratio decreases. A reasonable starting ratio could be 3.8 (the best ratio in our experiments). It is important to use a high concentration of CNT solution. Since the solution molar density should not vary significantly between batches of CNTs for a fixed mass, we recommend solution mass density of $17 \mu \mathrm{g} \mathrm{ml}^{-1}$. A solution of a higher concentration than this can reduce trapping yield as a more dense solution may result in denser networks with less or no bottlenecks. Lastly, using small drops of solution (e.g., $6 \mu \mathrm{l}$ ) that evaporate quickly (e.g., $\sim 30 \mathrm{~s}$ ) ensure that over deposition of CNTs on electrodes will not occur [6], over deposition being undesirable because of the reduction in the number of bottlenecks.

\section{Conclusion}

This study proved the feasibility of dielectrophoretically forming semiconductive networks for the construction of carbon nanotube field effect transistors out of a mixture of metallic and semiconductive nanotubes. Working DEP parameter sets have been identified, and an understanding of the factors that most impact the synthesis of these devices was obtained. The guidelines generated on the basis of this study could be useful to utilize this process on other nanotube samples. The encouraging, repeatable device characteristics make this trapping process an interesting approach for forming CNT-FETs.

\section{References}

[1] Zhao Q, Gan Z and Zhuang Q 2002 Electrochemical sensors based on carbon nanotubes Electroanalysis 14 1609-13

[2] Gruner G 2006 Carbon nanotube transistors for biosensing applications Anal. Bioanal. Chem. 384 322-35

[3] Kojima A, Hyon C K, Kamimura T, Maeda M and Matsumoto K 2005 Protein sensor using carbon nanotube field effect transistor Japan. J. Appl. Phys. 44 1596-8

[4] Dimaki M and Boggild P 2004 Dielectrophoresis of carbon nanotubes using microelectrodes: a numerical study Nanotechnology 15 1095-102

[5] Subramanian A, Dong L, Frutiger D and Nelson B J 2006 Shell engineering of carbon nanotube arrays by current driven breakdown Nanotechnology 2 901-4

[6] Li J, Zhang Q, Yang D and Tian J 2004 Fabrication of carbon nanotube field effect transistors by AC dielectrophoresis method Carbon 42 2263-7

[7] Chen R J, Bangsaruntip S, Drouvalakis K A, Kam N W S, Shim M, Li Y, Kim W, Utz P J and Dai H 2003 Noncovalent functionalization of carbon nanotubes for highly specific electronic biosensors Proc. Natl Acad. Sci. 100 4984-9

[8] Suehiro J, Zhou G and Hara M 2003 Fabrication of a carbon nanotube-based gas sensor using dielectrophoresis and its application for ammonia detection by impedance spectroscopy J. Phys. D: Appl. Phys. 36 L109-14

[9] Li J, Zhang Q, Peng N and Zhu Q 2005 Manipulation of carbon nanotubes using AC dielctrophoresis Appl. Phys. Lett. 86153116

[10] Tong J and Sun Y 2007 Toward carbon nanotube-based AFM cantilevers IEEE Trans. Nanotechnol. 6 519-27

[11] Seidel R, Graham A P, Unger E, Duesberg D S, Liebau M, Steinhoegl W, Kreupl F and Hoenlein W 2004 High-current nanotube transistors Nano Lett. 4 831-4

[12] Krupke R, Hennrich F, von Lohneysen H and Kappes M M 2003 Seperation of metallic from semiconducting single-walled carbon nanotubes Science 301 344-7

[13] Lutz T and Donovan K J 2005 Macroscopic scale seperation of metallic and semiconducting nanotubes by dielectrophoresis Carbon 43 2508-13

[14] Peng N, Zhang Q, Yuan S, Li H, Tian J and Chan L 2007 Current instability of carbon nanotube field effect transistors Nanotechnology $\mathbf{1 8} 424035$

[15] Chung J and Lee J 2003 Nanoscale gap fabrication and integration of carbon nanotubes by micromachining Sensors Actuators A 104 229-35

[16] Burke P J 2004 Nanodielectrophoresis: electronic nanotweezers Encycl. Nanosci. Nanotechnol. 6 623-41

[17] Tans S J, Verschueren A R M and Dekker C 1998 Room-temperature transistor based on a single carbon nanotube Nature 393 49-52

[18] Javey A, Guo J, Wang Q, Lundstrom M and Dai H 2003 Ballistic carbon nanotube field-effect transistors Nature 424 654-7

[19] Martel R, Schmidt T, Shea H R, Hertel T and Avouris P 1998 Single- and multi-wall carbon nanotube field-effect transistors Appl. Phys. Lett. 73 2447-9

[20] Seo H, Han C, Choi D, Kim K and Lee Y 2005 Controlled assembly of single SWNTs bundle using dielectrophoresis Microelectron. Eng. 81 83-9 